\title{
Cervical cancer risk perceptions, sexual risk behaviors and sexually transmitted infections among Bivalent Human Papillomavirus vaccinated and non- vaccinated young women in Uganda - 5 year follow up study
}

Edward Kumakech ${ }^{1,2^{*}}$, Sören Andersson ${ }^{2,3}$, Henry Wabinga ${ }^{4}$, Caroline Musubika ${ }^{5}$, Samuel Kirimunda ${ }^{6}$ and Vanja Berggren ${ }^{7}$

\begin{abstract}
Background: Previous studies were conflicting regarding the associations between HPV vaccination, cervical cancer risk perceptions, high-risk sexual behaviors and STIs. This study compared the HPV-vaccinated and non-vaccinated young women in Uganda regarding cervical cancer risk perceptions, high-risk sexual behaviors, syphilis and HIV infections 5 years after vaccine implementation.

Methods: This was a population-based comparative cross-sectional survey conducted in Uganda. The 438 participants were sexually active young women aged 15-24 years and mean age was 18.6 (SD 1.4). The majority (53.0\%) were HPV-vaccinated in 2008 without assessment of sexual activity prior to HPV vaccination. Upon verbal assessment of sexual activity at the time of follow-up, data were collected using a questionnaire and laboratory testing of blood samples for syphilis and HIV infections.
\end{abstract}

Results: There were no significant differences between the HPV-vaccinated and non-vaccinated groups regarding the prevalence of high-risk sexual behaviors, syphilis and HIV infections. Cervical cancer risk perceptions and age at sexual debut were nonetheless significantly lower among the vaccinated group compared to their non-vaccinated counterparts. However, HPV vaccination was not significantly associated to cervical cancer risk perceptions and early age at sexual debut in multivariate logistic regression analysis.

Conclusions: We found no associations between HPV vaccination, cervical cancer risk perceptions, high-risk sexual behaviors, syphilis and HIV infections among young women in Uganda 5 years after vaccine implementation. Young girls in the study population were found to be sexually active at a young age, affirming the importance of targeting girls of younger age for HPV vaccination.

Keywords: Bivalent Human Papillomavirus (HPV) vaccination, cervical cancer risk perceptions, sexual risk behaviors, Sexually transmitted infections, Young women, Uganda

\footnotetext{
* Correspondence: kumakeche@yahoo.com; Edward.kumakech@oru.se

${ }^{1}$ School of Biomedical Sciences, College of Health Sciences, Makerere

University, P.O. Box 7072, Kampala, Uganda

${ }^{2}$ School of Health and Medical Sciences, Örebro University, 70182 Örebro,

Sweden

Full list of author information is available at the end of the article
} 


\section{Background}

Cervical cancer (CC) is the third most common cancer among women globally in terms of 5-year prevalence (i.e. total cases identified over a 5-year period) with an estimated 527,624 new cases and 265,672 deaths in 2012 [1]. About $87 \%$ of the $\mathrm{CC}$ deaths occur in less developed countries. In East Africa, CC is the most common cancer and also the leading cause of cancer related death among women [1]. In Uganda, the age-standardized incidence rate for CC was estimated to stand at 44.4/ 100,000 women person years in 2012 which was one of the highest in the world [1].

An infection with sexually transmittable Human papillomavirus (HPV) is a necessary but not a sufficient cause of cervical cancer [2]. There are 14 so called high risk HPV (HR-HPV) genotypes associated with $\mathrm{CC}$ which include HPV 16/18 the antigens included in the currently available HPV vaccines [3]. Notably, $70 \%$ of cervical cancer cases are attributable to HPV16/18 alone, making the use of the two available HPV vaccines justifiable because both of them contain antigens for HPV16/18. In Uganda, HPV vaccination programme started in 2008 as a pilot project in two districts namely Ibanda and Nakasongola districts. The vaccine in use during the pilot phase was the bivalent HPV vaccine (Cervarix ${ }^{\oplus}$, GlaxoSmithKline, Belgium) which administered to girls aged 10 years or in primary school grade 5 in 3 doses at an interval of 0,1 and 6 months. The health workers take the vaccine to the targeted girls in schools as part of school health programme. In addition to school visits, community visits were also conducted to ensure eligible out of school girls also gets vaccinated. A phased approach to national rollout of HPV vaccination using the quadrivalent HPV vaccine (Gardasil, Merck MSD, USA) followed suit in 2010 initially in 12 districts and countrywide by 2014 . Nationally, the vaccine is administered to girls aged 9 years or in primary school grade 4 in 2 doses at an interval of 0 and 6 months. The delivery of the $\mathrm{HPV}$ vaccines to the girls is integrated into a bi-annual (every April and October) school health programme popularly known as child health days plus (CHDP) during which health workers visits schools to provide catchup vaccination and medication against intestinal worms. For out of school girls, the delivery of the vaccine is integrated into monthly community outreaches for immunization services delivery. HPV vaccination coverage survey conducted in the study area (Ibanda) around the period of the study indicated HPV vaccination coverage of $95 \%$ [4]. At the time of the study, the Uganda ministry of health had no written strategic plan for use of 9-valent HPV vaccine instead of or concurrently with bivalent and quadrivalent vaccines.

Important to note is that HPV vaccine alone would not completely prevent cervical cancer because the vaccine contains only 2 of the 14 HR-HPV types associated to CC. Secondly, it would be impossible to attain $100 \%$ vaccination coverage particularly in developing countries with weak health systems. Thirdly, potential epidemiological changes may occur as a result of HPV vaccination. The changes are most likely to arise because there are other HR-HPV types that are not covered by the current vaccines in developing countries (bivalent and quadrivalent HPV vaccines) and so it is possible that epidemiological changes indeed may occur by transitioning to disease caused by HR-HPV types not covered by HPV vaccination. Such discussions of the limitations of the currently available HPV vaccines are frequent in the literature [5-7]. The aforementioned discussions on the limitations of the currently available HPV vaccines that target HPV16/18 for CC prevention underscores the importance of simultaneously addressing other determinants of $\mathrm{CC}$ in order to optimize the benefits of HPV vaccination. Cervical cancer risk perceptions, high-risk sexual behaviors and other sexually transmitted infections (STIs) such as syphilis and HIV are some of the critical determinants of HPV infections and $\mathrm{CC}$ that needs to be addressed alongside HPV vaccinations $[8,9]$.

The status of high-risk sexual behaviors, syphilis and HIV infections in Uganda at the time of the study shows a decreasing trend in the prevalence of the above determinants among the 15-24 year-old young women from the pre-HPV vaccine introduction period to post-HPV vaccine period. For example, the percentage of the 1524 year-old young women who initiated sexual intercourse before the age of 15 decreased from $14.4 \%$ in 2005 to $10.9 \%$ in 2011 [10, 11]. Similarly, for syphilis, the percentage of the 15-24 year-old young women who were positive for syphilis decreased from $2.1 \%$ in 2005 to $1.4 \%$ in $2011[10,11]$. The same decreasing trend in the prevalence was also reported for HIV infection whereby the percentage of the 15-24 year-old young women who were positive for HIV decreased from 4.3\% in 2005 to $4.9 \%$ in 2011 [10, 11]. As for the trend of CC risk perceptions among young women in Uganda, previous studies specific to young women couldn't be found. Cervical cancer risk perceptions are often measured using a composite score calculated from a scale comprising of many items and therefore, point estimates also are not readily available for young women in Uganda.

Regarding the association between HPV vaccination and high risk sexual behaviors, previous studies were conflicting in their findings. Specifically, there were previous studies including in US and Canada that indicated no association between HPV vaccination, risk perceptions and high-risk sexual behaviors among young women [12-18]. For example, a study conducted among 18-30 year old Australian young women reported that attitudes to safe sexual behavior were the only factors 
significantly associated to vaccination status such that vaccinated women held more positive attitudes to practicing safe sexual behaviors [14]. Similarly, a short-range longitudinal study among 13-21 year-old HPV vaccinated young women from the US found no significant association between risk perceptions after HPV vaccination and high-risk sexual behaviors [18]. Other previous studies from the US and England reported that HPV vaccination was associated with reduction in safer sexual behaviors among youths but didn't lead to an increased sexual risk-taking behavior [19-21]. There were also previous studies that indicated that HPV vaccination was instead associated to safer sexual behaviors [4, 2224]. For example, a study conducted among Colombian young women found that HPV vaccination was inversely associated to perceived risk of HPV infection and perceived risk of cervical cancer, was not associated to high-risk sexual behaviors but instead the vaccinated women were more likely to use safer sexual behaviors such as consistent condoms use [23]. Similarly, other studies from the US reported that HPV vaccinated young women were more likely to use condoms than their unvaccinated counterparts [4] and also were no more likely to be treated for an STI than unvaccinated youth [24].

In view of the aforementioned conflicting previous studies on the association between HPV vaccination, CC risk perceptions, high-risk sexual behaviors and STIs, there is need for further research on the topic, more so from developing countries in Africa which carries the heaviest burden of CC. In this study, we compared the $\mathrm{HPV}$-vaccinated young women and their non-vaccinated counterparts in Uganda regarding CC risk perceptions, high-risk sexual behaviors and STIs 5 years after HPV vaccine implementation.

\section{Methods}

\section{Study design and area}

This was a population-based comparative cross sectional study. It was conducted in Ibanda district Uganda as part of a larger HPV16/18 (Cervarix ${ }^{\circledR}$ ) vaccine follow up study. Ibanda district was the district in Uganda where the first pilot bivalent HPV 16/18 vaccination project which targeted young women in primary school class 5 (P5) was implemented in 2008.

\section{Population and sample}

The study participants were HPV-vaccinated and nonvaccinated 15-24 year-old young women who were enrolled in secondary schools in Ibanda district. Young women were included in the study if they were sexually active and by 2008 were either in primary school class five (P5) in Ibanda district and were fully vaccinated with 3 doses of the bivalent HPV16/18 vaccine (vaccinated group) or were in primary school class six (P6) or higher in Ibanda district but were not vaccinated with bivalent HPV 16/18 vaccine (non-vaccinated group). Partially vaccinated young women were excluded from the study because they were expected to be very few as the 2008 $\mathrm{HPV}$ vaccination coverage in the area was above 95\% [25]. The 95\% HPV vaccination coverage implied that only $5 \%$ of the eligible young girls in the study area were either partially vaccinated (i.e. received 1 or 2 doses of the vaccine) or were not vaccinated at all. At the time of the 5-year follow in 2014, Uganda had not yet adopted the 2-dose regimen for HPV vaccination.

The high HPV vaccination coverage (95\%) among the 2008 cohort of girls also made it impractical to establish the required sample size for the non-vaccinated control arm of the study from the same 2008 cohort of girls. Therefore, two different cohorts of girls (i.e. 2006 and 2007 cohorts of the participating schools) were used for the non-vaccinated control group. This however posed the problem of differential age distribution between HPV vaccinated and non-vaccinated control groups.

The sample size was determined by power analysis calculation [26]. Any STI prevalence which was a categorical variable was used as the primary outcome variable for calculating the sample size. The calculation made use of the Normal approximation to the Binomial distribution. The prevalence of any STI in the non-vaccinated young women was assumed to be $10.7 \%$ because a previous study conducted in Uganda showed the prevalence of any STI among unvaccinated teenage young women with median age of 20 years was $10.7 \%$ [27]. The prevalence of any STI in the vaccinated group that would represent an important improvement from the HPV vaccination was assumed to be $1 \%$. And therefore, the proportions compared in the power analysis calculation were 0.107 and 0.01 and the sample size of 376 was reached at a power of $85 \%$ i.e. $85 \%$ probability of detecting such a difference, if it really existed, as statistically significant at $5 \%$ significance level. This was adjusted to 492 participants (i.e. 241vaccinated and 241 nonvaccinated young women) to cater for potential loss to follow up.

We performed multi-stage sampling procedure to select the study participants. We first developed a total list of senior secondary schools in Ibanda district where the 2008 cohort of HPV vaccinated young women and their non-vaccinated counterparts were expected to be studying at the time of the study. The total list comprised of 32 secondary schools. Being a school semester period, all the schools in the district were visited one after another by the research team. And young women found in senior secondary school classes three to six (i.e. S3-S6) were approached class by class, informed about the study, consented and screened for eligibility. Demographic data 
were obtained from all the young women approached. Appointment dates to visit Ruhoko health centre IV, a designated health facility within the district for data/ sample collection were agreed with the selected young women and their teachers. On the appointment day, vehicles were sent to the schools to facilitate transportation of the young women to and from the health facility in company of their teachers. Figure 1 shows the flow chart of the participants within the study.

\section{Data/sample collection}

Data/sample collection took place in 2014. At the health facility, data and samples were collected from consecutive young women reaching the facility until the required sample size was reached. The study procedures performed at the health facility included completing the CC questionnaire and collection of blood samples for HIV and syphilis testing.

\section{Questionnaire}

The standard questionnaire was used to collect data about the CC risk perceptions and sexual behaviors (Additional file 1). The questionnaire has two sections namely CC risk perceptions section and sexual behavior section.

The CC risk perceptions section comprised of 16 true or false items namely $\mathrm{CC}$ is the same as breast cancer, CC is caused by HPV, HPV is the same as HIV, HPV is transmitted from person to person through sexual intercourse, HPV vaccines when given to virgin young women protects against $\mathrm{CC}$, a virgin young girl should be receive 3 doses of HPV vaccines to be fully protected from $\mathrm{CC}$, women who missed $\mathrm{HPV}$ vaccine during their adolescence can still prevent $\mathrm{CC}$ by attending regular medical checkup/screening for $\mathrm{CC}, \mathrm{CC}$ is treatable if detected early, $\mathrm{CC}$ is more common among virgin women, women with many sexual partners have a higher chance of developing CC compared to those with one partner, HIV positive women have a higher chance of developing CC compared to HIV negative women, HPV doesn't infect men, circumcision of men doesn't reduce the chances of getting infected with HPV, having many sexual partners increases the chances of getting infected with HPV, condom use doesn't protect against HPV and HPV vaccines also protects against other STDs such as syphilis and gonorrhea. A score of 1 or 0 were awarded for each correct or wrong answer respectively. A sum of scores from the 16-items was obtained for each participant. The range for possible attainable scores was $0-16$. A higher score represent higher CC risk perceptions and a lower score represents lower $\mathrm{CC}$ risk perceptions. The risk perceptions section of the questionnaire was selfadministered under supervision of the study personnel.

Unlike the risk perceptions section questions which were scaled into a composite score, the sexual behavior section questions were used as independent outcome questions, and not scaled into a composite score. The sexual behavior section of the standard questionnaire comprised of 7-items namely age at first sexual intercourse, number of sexual partners in the previous 3 months, number of sexual partners in the previous 12 months, number of sexual partners in the previous 4 years, number of sexual partners in a lifetime, history of STD syndrome (whether she has ever experienced

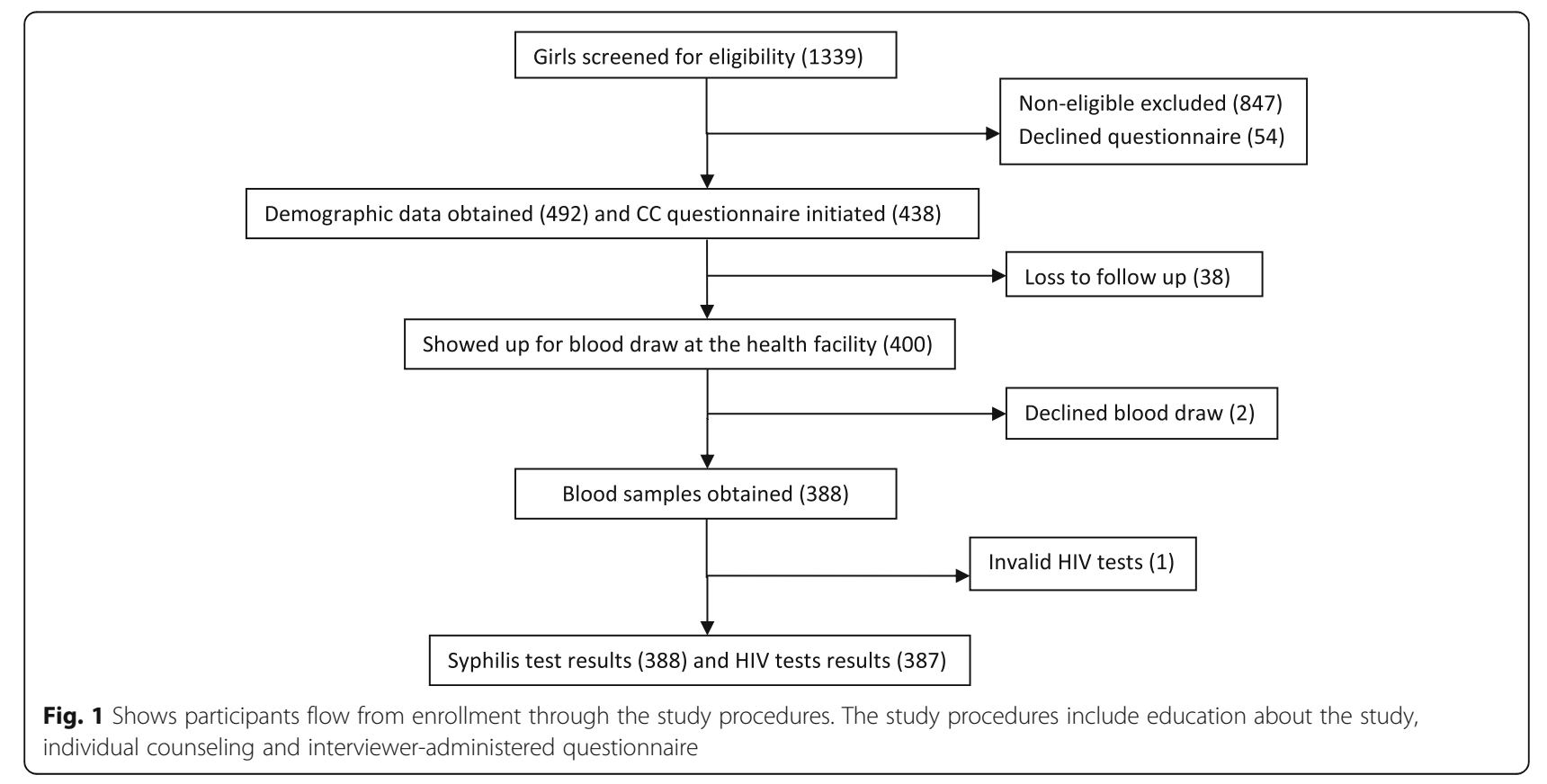


any abnormal vaginal discharge or lower abdominal pain or genital ulcer or groin swelling, warts, vaginal discharge in her life) and level of condom use (whether never, rarely, sometime, often and always). The sexual behavior section of the questionnaire was intervieweradministered to ensure proper phrasing or probing for correct answers and was coupled with health history taking, physical examination and prescription of treatment for those found with health problems.

Both the risk perception and sexual behavior sections of the survey instrument were created by the Study Investigators specifically for the study. Face validity of the instrument was achieved by sending the questionnaire to a sample of 5 experts on HPV vaccination and all of them responded back with the judgement that the scale appears to be a good measure of CC risk perceptions. Armed with the criteria (Additional file 2) for adolescent $\mathrm{CC}$ risk perception, the same sample of experts examined the scale for adolescent $\mathrm{CC}$ risk perception and all came with the judgement the scale meet the criteria can legitimately be defined as adolescent CC risk perception scale. The reliability index Cronbach's alpha for the CC risk perception section of the questionnaire which was a scale was 0.67 after accounting for reversely-worded items which was reasonably strong reliability of the scale to measure $\mathrm{CC}$ risk perception.

\section{HIV testing}

Fresh venous blood samples drawn from the cubical vein using EDTA vacutainer by two trained/experienced laboratory technicians were used for the HIV tests. HIV-1 testing was performed using first the Determine rapid test (Abbot Diagnostics) [28]. The samples that were not reactive were considered HIV-negative. Otherwise, the Statpak rapid tests (ChemoBio Diagnostics Systems) were used to confirm HIV positivity. In case of disagreement between the 2 tests, a tie-breaker test, the Unigold rapid test (Orgenics), was used. This was the recommended national HIV testing algorithm in Uganda at the time of the study. An independent laboratory technician performed all the HIV testing from Kiwoko health centre IV laboratory in Ibanda district. Young women whose HIV test results turned out to be positive were linked to HIV/AIDS treatment and care program available at the same health facility where all the HIV tests were performed.

\section{Syphilis testing}

Parts of the same venous blood samples drawn for the HIV tests were used for the Syphilis tests. Serum extracted from the venous blood from the laboratory at the study site were kept and transported in liquid nitrogen from the field site to Immunology laboratory at Makerere University College of Health Sciences where they were immediately transferred to minus $80 \mathrm{C}$ freezers until testing. The liquid nitrogen tanks ensured cold chain for samples during transportation from fieldwork site to the laboratory. Syphilis tests were performed using a commercially available kit, Human Treponema Pallidum Hemagglutination Assay (TPHA) liquid GmBH, Wiesbaden, German.). The TPHA test is a qualitative microhemagglutination test for the presence of IgG and IgM antibodies to Treponema pallidum, the causative agent for syphilis, in human serum and EDTA plasma. The tests were performed according to the manufacturer's protocol. Women with positive TPHA test results were referred to clinician for treatment. All the Syphilis tests were performed by yet another independent laboratory technician who wasn't part of the blood draw team from the Immunology laboratory at Makerere University College of Health Sciences Kampala Uganda.

\section{Statistical analysis}

Data were entered into Statistical Package for Social Sciences (SPSS) version 22.0 for analysis. The HPVvaccinated and non-vaccinated groups were compared for the demographic data, CC risk perceptions, sexual behaviors and STI prevalence using Pearson's' chi-square $\left(x^{2}\right)$ or Fisher's exact tests for categorical variables which also generated $p$ values, odd ratio (OR) and 95\% confidence interval. Continuous variables were compared using independent sample t-test which also provided $p$ values. Additionally, using early age at sexual debut $(<16$ years vs $16+$ years $)$ as the categorical outcome variable, logistic multivariate regression was performed to further examine the association between HPV vaccination and early age at sexual debut after controlling for all other covariates (age, educational level and CC risk perceptions). These covariate included in the logistic multivariate regression were the variables that were significantly associated to early age at sexual debut from the bivariate analysis and were theoretically relevant. Although Uganda national age at sexual debut among females was 15 years $[10,11]$, in this study, early age at sexual debut cut off was put at $<16$ because no study participant was aged $<15$ years.

\section{Results}

The age range of the 438 study participants which comprised of 50.4\% HPV-vaccinated young women was 1524 years and mean age was 18.6 (SD 1.4). All the participants were female students attending secondary school in Ibanda district, the study area in rural southwestern Uganda. All the participants were non-smokers, with neither history of pregnancy, nor child birth, nor abortion nor hormonal contraceptive use. As shown in participants flow schema 1 , non-eligibility rate was $63 \%$ and the major reason for non-eligibility was sexual inactivity. 
As shown in schema 1, of the 492 eligible young women whose demographic data were obtained for the study, 54 declined responding to the $\mathrm{CC}$ questionnaire. Of the 438 who initiated responding to the $\mathrm{CC}$ questionnaire, 38 didn't turned up to the health facility for blood draw. Of the 400 who turned up for blood draw, 2 declined blood draw and finally of the 388 blood samples obtained for HIV tests, 1 was invalid for HIV test. The demographic characteristics of the 54 young women who declined the $\mathrm{CC}$ questionnaire plus the 38 young women who didn't show up for blood draw and the 2 young women who declined blood draw were not significantly different from the rest of the study participants who completed the study (statistics not presented here).

\section{Demographic characteristics of the study participants}

Table 1 shows the demographic characteristics of the study participants. With the exception of age, educational level and ethnic tribe, there were no statistically significant differences between HPV-vaccinated and non-vaccinated control groups regarding their demographic characteristics. As shown in Table 1, the non-vaccinated control group was significantly older but also began sexual activity at significantly older age than the HPV-vaccinated group. The age difference between vaccinated and control groups is attributable to the educational level targeted for HPV vaccination in 2008 which was primary school class five irrespective of their age. As shown in Table 1, the HPV-vaccinated young women were significantly younger in age, mostly Ankole by ethnic tribe and mostly from S1-S4 classes compared to the non-vaccinated control young women.

\section{Cervical cancer risk perceptions}

As shown in Table 2 (bivariate analysis of the association between cervical cancer risk perceptions and HPV vaccination), the overall cervical cancer risk perceptions among the HPV-vaccinated young women were significantly lower than that among the non-vaccinated young women.

In multivariate analysis for the association between low cervical cancer risk perception and set predictors (tribe, religion, address, age at sexual debut and HPV vaccination) as shown in Table 3 , HPV vaccination was not significant useful predictor of low cervical cancer risk perception.

\section{High-risk sexual behaviors}

In bivariate analysis (Table 4), there were no significant differences between the HPV-vaccinated and nonvaccinated young women regarding high-risk sexual behaviors with exception of age at sexual debut. The mean age at sexual debut among the HPV-vaccinated young women was significantly lower than that among their non-vaccinated counterparts [15.5 vs. 16.1, p 0.018]. Low at age at sexual debut was used as one of the markers of high-risk sexual behavior among the young girls in this study.

Table 1 Demographic characteristics by HPV vaccination status of the participants

\begin{tabular}{|c|c|c|c|c|}
\hline \multirow{3}{*}{ Demographic } & \multirow{3}{*}{$\begin{array}{l}\text { Total } \\
\mathrm{N}\end{array}$} & \multicolumn{2}{|l|}{ Study groups } & \multirow{3}{*}{$p$-value } \\
\hline & & HPV-vaccinated & Non-vaccinated & \\
\hline & & {$[f(\%)]$} & {$[f(\%)]$} & \\
\hline Age in years [mean (sd)] & 18.6(sd1.4) & 18.1(sd1.2) & 19.1(sd1.4) & $0.000^{*}$ \\
\hline \multicolumn{5}{|l|}{ Age group } \\
\hline$\cdot 18+$ years & 373 & $373(44.5)$ & $207(55.5)$ & \multirow[t]{2}{*}{$0.000^{*}$} \\
\hline$\cdot<18$ years & 110 & $85(77.3)$ & $25(22.7)$ & \\
\hline \multicolumn{5}{|l|}{ Address } \\
\hline - Urban & 343 & $170(67.2)$ & 173(72.4) & \multirow[t]{2}{*}{0.210} \\
\hline • Rural & 149 & 83(32.8) & $66(27.6)$ & \\
\hline \multicolumn{5}{|l|}{ Ethnic tribe } \\
\hline - Ankole & 358 & $210(58.7)$ & 148(41.3) & \multirow[t]{2}{*}{$0.000^{*}$} \\
\hline - Others & 97 & $31(32.0)$ & $66(68.0)$ & \\
\hline \multicolumn{5}{|l|}{ Religion } \\
\hline - Christians & 448 & 238(53.1) & 210(46.9) & \multirow[t]{2}{*}{0.670} \\
\hline • Muslims & 5 & $2(40.0)$ & $3(60.0)$ & \\
\hline \multicolumn{5}{|l|}{ Education level } \\
\hline$\cdot$-S1-S4 & 348 & $251(72.1)$ & $97(27.9)$ & \multirow[t]{2}{*}{$0.000^{*}$} \\
\hline$\cdot$ S5-S6 & 134 & $1(0.7)$ & 133(99.3) & \\
\hline
\end{tabular}

$\mathrm{N}$ is total sample size; $\mathrm{f}$ is the frequency; (\%) is the percentage; Sd is standard deviation; S1-S4 is senior 1 to senior 4 class; S5-S6 is senior 5 to senior 6 class; ${ }^{*}$ is statistically significant $p$ value 
Table 2 Bivariate analysis of the Association between Cervical cancer risk perceptions and HPV vaccination

\begin{tabular}{llll}
\hline Item & $\begin{array}{l}\text { Total } \\
N\end{array}$ & $\begin{array}{l}\text { HPV-vaccinated } \\
{[f(\%)]}\end{array}$ & $\begin{array}{l}\text { Non-vaccinated } \\
{[f(\%)]}\end{array}$ \\
$\begin{array}{l}\text { Cervical cancer is the same } \\
\text { as breast cancer }\end{array}$ & & \\
• True & 82 & $45(19.4)$ & $37(18.0)$ \\
• False & 356 & $187(80.6)$ & $69(82.0)$ \\
Cervical cancer is caused by HPV & & & $22(10.7)$ \\
• False & 48 & $26(11.3)$ & $184(89.3)$ \\
• True & 389 & $205(88.7)$ & 0.794 \\
HPV is the same as HIV & & $38(16.6)$ & $35(17.0)$ \\
• True & 73 & $191(83.4)$ & $171(83.0)$ \\
• False & 362 & & 1.000
\end{tabular}

HPV is transmitted from person

to person through sexual intercourse

- False 42

- True

HPV vaccines when given to young

girls protect them against cervical cancer

- False

- True

A young girl should be given 3 doses of HPV vaccines to be fully protected against cervical cancer

$$
\text { - False }
$$

- True

Women who missed HPV vaccinations during their adolescence can still prevent cervical cancer by attending regular checkup

$$
\text { - False }
$$

$$
\text { - True }
$$

Cervical cancer is treatable if detected early

- False

Cervical cancer is more common among women who have never had sexual intercourse in their lifetime

$$
\begin{aligned}
& \text { - True } \\
& \text { - False }
\end{aligned}
$$

Women with many sexual partners have a higher chance of developing cervical cancer than those with fewer partners

$$
\text { - False }
$$$$
\text { - True }
$$

More of the HIV positive women develop cervical cancer compared to the HIV negative women

$$
\text { - False }
$$

- True
192(93.2)

19(9.2)

0.044

187(90.8)

20(9.7)

0.394

213(93.0)

186(90.3)

48(23.3)

0.610

169(74.1)

158(76.7)

6(2.9)

0.583

219(95.6)

200(97.1)

163(79.1)

16(7.8)

0.035

$198(85.3)$

190(92.2)
46(22.3)

160(77.7) 
Table 2 Bivariate analysis of the Association between Cervical cancer risk perceptions and HPV vaccination (Continued)

\begin{tabular}{|c|c|c|c|c|}
\hline \multicolumn{5}{|l|}{ HPV doesn't infect men } \\
\hline - True & 297 & 159(68.8) & 138(67.0) & \multirow[t]{2}{*}{0.757} \\
\hline - False & 140 & $72(31.2)$ & 68(33.0) & \\
\hline \multicolumn{5}{|c|}{$\begin{array}{l}\text { Circumcision of men doesn't } \\
\text { reduce the chances of } \\
\text { getting infected with HPV }\end{array}$} \\
\hline - True & 214 & 114(49.8) & $100(48.5)$ & \multirow[t]{2}{*}{0.871} \\
\hline - False & 221 & 115(50.2) & $106(51.5)$ & \\
\hline \multicolumn{5}{|c|}{$\begin{array}{l}\text { Having many sexual partners } \\
\text { increases the chances of } \\
\text { getting infected with HPV }\end{array}$} \\
\hline - False & 43 & $23(10.0)$ & $20(9.7)$ & \multirow[t]{2}{*}{1.000} \\
\hline - True & 394 & 208(90.0) & 186(90.3) & \\
\hline \multicolumn{5}{|c|}{$\begin{array}{l}\text { Condom use doesn't protect } \\
\text { against HPV }\end{array}$} \\
\hline - False & 217 & $104(45.4)$ & \multirow[t]{2}{*}{ 113(54.9) } & 0.061 \\
\hline - True & 218 & $125(54.6)$ & & $93(45.1)$ \\
\hline \multicolumn{5}{|c|}{$\begin{array}{l}\text { HPV vaccines also protect against } \\
\text { other STDs such as syphilis } \\
\text { and Gonorrhea }\end{array}$} \\
\hline - True & 177 & $99(43.2)$ & $78(37.9)$ & \multirow[t]{2}{*}{0.298} \\
\hline - False & 258 & 130(56.8) & $128(62.1)$ & \\
\hline \multicolumn{5}{|l|}{ CC risk perception } \\
\hline - Overall mean score (sd) & $12.1(\mathrm{sd} 2.1)$ & $11.9(\mathrm{sd} 2.3)$ & 12.3(sd 1.8) & $0.021^{*}$ \\
\hline
\end{tabular}

$\mathrm{N}$ is total sample size; CC is cervical cancer; $\mathrm{f}$ is the frequency; (\%) is the percentage; $\mathrm{Sd}$ is standard deviation; ${ }^{*}$ is statistically significant $p$ value

\section{Factors associated to early age at sexual debut among the participants}

As shown in Table 4, at bivariate analysis level, four factors were significantly associated to early age at sexual debut namely having an age less than 16 years, lower

Table 3 Multivariate Analysis of Predictors for low CC risk perceptions among the young women

\begin{tabular}{lll}
\hline & $p$ & OR 95\% Cl \\
\hline Tribe & 0.653 & $0.88(0.51-1.53)$ \\
• Ankole & ref & \\
• Others & & \\
Religion & 0.609 & $1.84(0.18-19.08)$ \\
• Christians & ref \\
• Muslims & \\
Address & 0.853 & \\
• Urban & ref & \\
• Rural & 0.108 & \\
Age at sexual debut & $0.94(0.64-1.71)$ \\
HPV vaccination status & & \\
• HPV-vaccinated & 0.638 & $1.14(0.66-1.97)$ \\
- -vaccinated & ref & \\
\hline
\end{tabular}

Controlled variables were age and education level. $\mathrm{p}$ is $p$-value; OR is Odd ratio; $95 \% \mathrm{Cl}$ is $95 \%$ Confidence interval; * is statistically significant $p$-value educational level [i.e. being senior secondary class one to four (S1-S4)], lower cervical cancer risk perceptions and $\mathrm{HPV}$ vaccination.

In multivariate logistic regression analysis level shown in Table 5, it was only age that was a significant predictor of early age at sexual debut [p 0.000, exp.(B) 0.5]. The rest of the factors in the model including HPV vaccination, low education level and low CC risk perceptions were not significant predictors of early age at sexual debut among the participants.

\section{Prevalence of syphilis and HIV infections}

In bivariate analysis (Table 4), there were no statistically significant differences between the HPV-vaccinated and non-vaccinated young women regarding the prevalence of syphilis and HIV infections. Syphilis and HIV infections were used as some of the bio-markers of high-risk sexual behaviors among the young women in this study.

\section{Discussion}

The HPV-vaccinated young women of our sample were significantly younger in age and were mostly from the lower senior secondary educational level (S1-S4) compared to their non-vaccinated counterparts. This was attributable to the participants sampling strategy used in the study which targeted the 2008 cohort of HPV- 
Table 4 Bivariate analysis of the Association between sexual behaviors, sexually transmitted infections and HPV vaccination status of the participants

\begin{tabular}{|c|c|c|c|c|}
\hline \multirow{3}{*}{ Item } & \multirow{3}{*}{$\begin{array}{l}\text { Total } \\
\mathrm{N}\end{array}$} & \multicolumn{2}{|l|}{ Study groups } & \multirow{3}{*}{$p$} \\
\hline & & HPV-vaccinated & Non-vaccinated & \\
\hline & & {$[f(\%)]$} & {$[f(\%)]$} & \\
\hline Age at sexual debut (mean in years) & $15.9(\mathrm{sd} 2.9)$ & $15.5(\mathrm{sd} 2.7)$ & $16.1(\mathrm{sd} 3.1)$ & $0.018^{*}$ \\
\hline \multicolumn{5}{|l|}{ Age group at sexual debut } \\
\hline$\cdot<16$ years & 197 & $68(34.5)$ & $129(65.5)$ & \multirow[t]{2}{*}{0.073} \\
\hline$\cdot 16+$ years & 184 & $47(25.5)$ & $137(74.5)$ & \\
\hline \multicolumn{5}{|l|}{$\begin{array}{l}\text { Number of sexual partners } \\
\text { in previous } 3 \text { months }\end{array}$} \\
\hline$\cdot 1+$ sexual partners & 206 & 113(56.8) & $93(50.3)$ & \multirow[t]{2}{*}{0.239} \\
\hline$\cdot 0$ & 178 & $86(43.2)$ & $92(49.7)$ & \\
\hline \multicolumn{5}{|l|}{$\begin{array}{l}\text { Number of sexual partners } \\
\text { in previous } 1 \text { year }\end{array}$} \\
\hline$\cdot 1+$ sexual partners & 282 & $150(75.0)$ & $132(71.4)$ & \multirow[t]{2}{*}{0.488} \\
\hline$\cdot 0$ & 103 & $50(25.0)$ & $53(28.6)$ & \\
\hline \multicolumn{5}{|l|}{$\begin{array}{l}\text { Number of sexual partners } \\
\text { in previous } 4 \text { years }\end{array}$} \\
\hline$\cdot 1+$ sexual partners & 297 & 153(76.5) & 144(77.8) & \multirow[t]{2}{*}{0.849} \\
\hline$\cdot 0$ & 88 & $47(23.5)$ & $41(22.2)$ & \\
\hline \multicolumn{5}{|l|}{$\begin{array}{l}\text { Number of sexual partners } \\
\text { in a lifetime }\end{array}$} \\
\hline$\cdot 2+$ sexual partners & 65 & $31(15.5)$ & $34(18.5)$ & \multirow[t]{2}{*}{0.521} \\
\hline - 1 partner & 319 & 169(84.5) & 150(81.5) & \\
\hline \multicolumn{5}{|l|}{ Condom use } \\
\hline - Never used & 229 & $121(60.2)$ & $108(58.1)$ & \multirow[t]{2}{*}{0.746} \\
\hline • Ever used & 158 & $80(39.8)$ & $78(41.9)$ & \\
\hline \multicolumn{5}{|l|}{ History of STD syndrome } \\
\hline - Positive & 58 & 28(13.9) & $30(16.1)$ & \multirow[t]{2}{*}{0.643} \\
\hline - Negative & 329 & 173(86.1) & 156(83.9) & \\
\hline \multicolumn{5}{|l|}{ TPHA (syphilis) test result } \\
\hline - Positive & 4 & $1(0.5)$ & $3(1.6)$ & \multirow[t]{2}{*}{0.351} \\
\hline - Negative & 384 & 202(99.5) & 182(98.4) & \\
\hline \multicolumn{5}{|l|}{ HIV test result } \\
\hline - Positive & 7 & $2(1.0)$ & $5(2.7)$ & 0.264 \\
\hline - Negative & 380 & 201(99.0) & & 179(97.3) \\
\hline
\end{tabular}

Sd is standard deviation; TPHA is Treponema pallidum hemagglutination test for syphilis; STI is sexually transmitted infections; STD is Sexually transmitted diseases; $\mathrm{N}$ is total sample size; $\mathrm{f}$ is frequency; (\%) is percentage; $\mathrm{p}$ is $p$-value at $5 \%$ significance level; ${ }^{*}$ is statistically significant $p$-value

vaccinated young women who were expected to be younger and in the lower educational level compared to their non-vaccinated counterparts recruited mostly from the higher senior secondary class five to six (S5-S6).

The ethnic tribal differences between the HPVvaccinated and non-vaccinated groups observed in the sample for this study could also be attributed to the fact that the 2008 pilot HPV vaccine project in Uganda was implemented in Ibanda district (the study area), a district predominantly occupied by people of Ankole ethnic tribe and hence the HPV-vaccinated young women were more likely to belong to the Ankole ethnic tribe. Notably, the selection of Ibanda district and hence high participation of young women of Ankole ethnic tribe in HPV vaccine rollout program and the study was neither based on HPV and cervical cancer disease burden differences nor cultural differences between ethnic tribes with regard to sexual activity that may impact these findings.

Interestingly, the $\mathrm{CC}$ risk perceptions demonstrated by the HPV-vaccinated young women in this study were 
Table 5 Multivariate Analysis of Predictors of early age at sexual debut

\begin{tabular}{|c|c|c|}
\hline & $\mathrm{p}$ & OR 95\% Cl \\
\hline \multicolumn{3}{|l|}{ Tribe } \\
\hline - Ankole & 0.664 & $1.14(0.63-2.07)$ \\
\hline - Others & ref & \\
\hline \multicolumn{3}{|l|}{ Religion } \\
\hline - Christians & 0.298 & $0.33(0.04-2.64)$ \\
\hline - Muslims & ref & \\
\hline \multicolumn{3}{|l|}{ Address } \\
\hline - Urban & 0.680 & $0.90(0.54-1.50)$ \\
\hline • Rural & ref & \\
\hline CC risk perception score & $0.010^{*}$ & $0.86(0.77-0.97)$ \\
\hline \multicolumn{3}{|l|}{ HPV vaccination status } \\
\hline - HPV-vaccinated & 0.869 & $0.95(0.54-1.68)$ \\
\hline - Non-vaccinated & ref & \\
\hline
\end{tabular}

Variables controlled for were age and education level. $p$ is $p$-value; OR is Odd ratio; $95 \% \mathrm{Cl}$ is $95 \%$ Confidence interval; * is statistically significant $p$-value

significantly lower than that for the non-vaccinated control group although it wasn't significantly associated to high-risk sexual behaviors. This finding was consistent with previous studies from Colombia and US that reported an inverse association between HPV vaccination and perceived risk of CC $[18,23]$ in bivariate but also not in multivariate analysis. In our sample, the CC risk perceptions differences between the HPV-vaccinated and non-vaccinated groups were attributable to misconceptions such as HPV vaccines doesn't protect against CC, multiple sexual partners doesn't increase the risk of CC and HIV infection doesn't increase the risk of CC which were significantly more prevalent among the HPVvaccinated group compared to their non-vaccinated counterparts. This finding underscores the importance of educating young women about the risk factors for CC in addition to HPV vaccines as part of vaccine implementation.

With exception of age at sexual debut (data in Table 4), we found no significant differences between HPVvaccinated young women and their non-vaccinated counterparts regarding high-risk sexual behaviors. Our findings of no association between HPV vaccination and other high-risk sexual behaviors rather than age at sexual debut contradicts with several previous studies that reported that HPV-vaccinated young women were more likely to experience sexual intercourse [14] or were more likely to have positive attitudes toward maintaining safe sexual health [22] or were more likely to use safer sexual behaviors such as consistent condoms use [23] or were more likely to use condoms [4] or were no more likely to be treated for an STI than the unvaccinated youth [24]. On the other hand, our finding of no association between HPV vaccination and other high-risk sexual behaviors concurs with some previous studies including in US and Canada that similarly indicated no association between HPV vaccination and high-risk sexual behaviors among young women [12-18]. In view of the above, our finding in agreement with more recent previous studies doesn't support the possibility of sexual disinhibition among young women following HPV vaccination.

Our finding regarding the association of HPV vaccination with early age sexual debut in bivariate analysis although not significant in multivariate analysis contracts with a number of previous studies from other parts of the world that reported no association between HPV vaccination and early age at sexual debut in both bivariate and multivariate analyses [\&, 11, 12, 14, 22]. In our sample, the association between HPV vaccination and early age at sexual debut was probably confounded by other covariates among the participants such that in multivariate model containing other covariates, HPV vaccination was found to be not a significant predictor of early age at sexual debut but age. This could imply that young women of younger generation (vaccinated group) starts sexual activity at an earlier age than young women of older generation (non-vaccinated group), emphasizing the importance of initiating HPV vaccination of young women at a younger age than 15 years before they start sexual activity.

The HPV-vaccinated young women in our sample didn't differ significantly from their non-vaccinated counterparts regarding the history of STD syndrome and the prevalence of both syphilis and HIV infections. These findings from our study were consistent with previous studies from the US and Sweden that both reported no significant differences between HPVvaccinated and their non-vaccinated counterparts regarding STI prevalence rates $[12,14]$. Since STIs are often used as objective biomarker of safer sexual behaviors, our findings in line with previous studies regarding no association between HPV vaccination and STIs further suggest that HPV vaccination doesn't have detrimental effect on sexual health of young women.

The strengths of our study include being one of the first HPV vaccine follow up studies conducted from developing country in Africa with high burden of HPVrelated cancers to empirically examine the association between HPV vaccination, CC risk perceptions and high-risk sexual behaviors using a population-based comparative cross sectional survey design. We assessed for sexual behaviors both subjectively using a questionnaire and objectively by performing laboratory tests for syphilis and HIV infections which were the respectable biomarkers of high-risk sexual behaviors used in previous related studies among young women [12, 14]. And we made use of non-vaccinated control group and 
multivariate logistic regression to control for extraneous variables.

A weakness of our study was that some of the data were self-reported and hence subject to reporting bias. We minimized on reporting bias by using a standard questionnaire and ensuring that the sexual behavior section of the questionnaire was interviewer-administered which provided opportunities for clarification on questions and probing. Another potential weakness was the fact that we studied a largely rural in-school young women population shortly after their sexual debut and hence our results may not be widely applicable to a more diverse population.

Further research on this topic in developing country particularly Africa should be a long term HPV vaccine follow up of the young women for cervical cancer secondary prevention outcomes such as attitudes and uptake of cervical cancer screening.

\section{Conclusions}

We found that CC risk perceptions and age at sexual debut were both significantly lower among the HPV-vaccinated young women compared to their non-vaccinated counterparts in Uganda 5 years after vaccine implementation. However, HPV vaccination was neither associated to lower cervical cancer risk perceptions nor early age at sexual debut in multivariate analysis. Other high-risk sexual behaviors were rampant among both groups although the differences were not statistically significant. Our findings don't support the notion that HPV vaccination is associated with high-risk sexual behaviors. Importantly, we found that young women in study area in rural Uganda were sexually active at a younger age and are engaged in high-risk sexual behaviors, affirming the importance of targeting young women of younger age for HPV vaccination.

\section{Additional file}

Additional file 1: Questionnaire. (DOCX $52 \mathrm{~kb}$ )

Additional file 2: STROBE Statement-checklist of items that should be included in reports of observational studies. (DOC $109 \mathrm{~kb}$ )

\section{Abbreviations \\ CC: Cervical cancer; EDTA: Ethylene diamine tetra-acetic acid; HIV: Human Immunodeficiency Virus; HPV: Human Papillomavirus; IgG: Immunoglobulin G; IgM: Immunoglobulin M; P5: Primary school class 5; P6: Primary school class 6; S3: Senior secondary school class 3; S4: Senior secondary school class 4; S5: Senior secondary school class 5; S6: Senior secondary school class 6; SD: Standard deviation; STDs: Sexually transmitted disease(s); STIs: Sexually Transmitted Infection(s); TPHA: Treponema Pallidum Hemagglutination}

\section{Acknowledgments}

We thank Swedish International Development Cooperation Agency (SIDA) for funding the study. We also thank the participants for accepting to participate in the study.

\section{Funding}

We are grateful to Swedish International Development Agency (SIDA) for providing funding for the research project.

\section{Availability of data and materials}

The raw data and materials are available and can be obtained from the Institutional Review Board (IRB) of Makerere University College of Health Sciences in Uganda.

\section{Authors' contributions}

EK conceived of the study, and participated in its designing, collecting data, analysis and writing the manuscript. SA participated in conceiving the study and in its design, supervising data collection and reviewing the manuscript. CM and SK participated in collecting blood samples and laboratory analysis for HIV and syphilis infections in Uganda and reviewing the manuscript. HW participated in designing the study, supervising data collection and reviewing the manuscript. VB participated in conceiving the study and in its design and reviewing the manuscript. All authors read and approved of the final manuscript.

\section{Authors' information}

EK, a holder of BSN, MSN/MPH is a doctoral student at Makerere University Uganda in collaboration with Örebro University Sweden.

$\mathrm{SA}$, a holder of MD, PhD is an Associate Professor and the Head, Department of Laboratory Medicine at Örebro University Hospital Sweden and Örebro University Sweden.

HW, a holder of MD, PhD is a Professor of Pathology and Director of Kampala Cancer Registry at Makerere University Uganda.

CM, a holder of MLS, MSC is a Laboratory Scientist in the Department of Medical Microbiology, Immunology laboratory, Makerere University Uganda. SK, a holder of MLS, MSc is a Laboratory Scientist in the Department of Medical Microbiology, Immunology laboratory, Makerere University Uganda. $\mathrm{VB}$, a holder of MPH, PhD is an Associate Professor in the Department of Health Sciences, Lund University Sweden

\section{Competing interests}

All the authors declared neither financial nor non-financial conflict of interests.

\section{Consent for publication}

In Uganda, the informed consent and assent provided by the study respondents / subjects usually is inclusive of the consent to publish the data/findings.

\section{Ethics approval and consent to participate}

The ethical clearance for this study was obtained from the Institutional Review Board (IRB) of Makerere University College of Health Sciences in Uganda. Additional research clearance was obtained from Uganda National Council for Science and Technology. Informed consent were obtained from each participant aged 18 years and above before inclusion into the study. For participants below the age 18 years, assent was obtained from them plus a proxy informed consent from their school teachers because all the participants were in school full board at the time of the study. Additionally, all participants were provided free of charge transportation to and from the health facility for sample collection. And at the health facility, all participants were served a soft drink and were provided the opportunity to receive healthcare services if indicated.

\section{Publisher's Note}

Springer Nature remains neutral with regard to jurisdictional claims in published maps and institutional affiliations.

\section{Author details}

${ }^{1}$ School of Biomedical Sciences, College of Health Sciences, Makerere University, P.O. Box 7072, Kampala, Uganda. ${ }^{2}$ School of Health and Medical Sciences, Örebro University, 70182 Örebro, Sweden. ${ }^{3}$ Department of Laboratory Medicine, Örebro University Hospital, 70362 Örebro, Sweden. ${ }^{4}$ Department of Pathology, Kampala Cancer Registry, Makerere University, P.O. Box 7072, Kampala, Uganda. ${ }^{5}$ Department of Medical Microbiology, Immunology Laboratory, Makerere University, P.O. Box 7072, Kampala, Uganda. ${ }^{6}$ Department of Medical Microbiology, Immunology laboratory, Makerere University, P.O. Box 7072, Kampala, Uganda. ${ }^{7}$ Department of Health Sciences, Lund University, 22100 Lund, Sweden. 
Received: 19 November 2015 Accepted: 24 May 2017

Published online: 02 June 2017

\section{References}

1. International Agency for Research on Cancer (IARC) and WHO: Globocan 2012: Estimated cancer incidence, mortality and prevalence worldwide in 2012. Available at http://globocan.iarc.fr/. Accessed 1 Nov 2013.

2. Walboomers JM, Jacobs MV, Manos MM, Bosch FX, Kummer JA, Shah KV, et al. Human papillomavirus is a necessary cause of invasive cervical cancer worldwide. J Pathol. 1999;189(1):12-9.

3. Munoz N, Castellsague X, de Gonzalez AB, Gissmann L. Chapter 1: HPV in the etiology of human cancer. Vaccine. 2006;24S3:S1-S10.

4. Liddon NC, Leichliter JS, Markowitz LE. Human papillomavirus vaccine and sexual behavior among adolescent and young women. Am J Prev Med. 2012:42:44.

5. Chen Z, Schiffman M, Herrero R, DeSalle R, Anastos K, et al. Evolution and Taxonomic Classification of Human Papillomavirus 16 (HPV16)-Related Variant Genomes: HPV31, HPV33, HPV35, HPV52, HPV58 and HPV67. PLoS One. 2011;6(5):e20183. doi:10.1371/journal.pone.0020183.

6. Chen Z, Schiffman M, Herrero R, DeSalle R, Anastos K, et al. Evolution and Taxonomic Classification of Alphapapillomavirus 7 Complete Genomes: HPV18, HPV39, HPV45, HPV59, HPV68 and HPV70. PLoS One. 2013:8(8): e72565. doi:10.1371/journal.pone.0072565.

7. Muñoz N, Bosch FX, Castellsagué X, Díaz M, De Sanjose S, Hammouda D, et al. Against which human papillomavirus types shall we vaccinate and screen, the international perspective. Int J Cancer. 2004;111:278-85.

8. Odida M, de Sanjosé S, Quint W, Bosch FX, Klaustermeier J, Weiderpass E. Human Papillomavirus type distribution in invasive cervical cancer in Uganda. BMC Infect Dis. 2008;8:85.

9. Banura C, Mirembe FM, Katahoire AR, Namujju PB, Mbonye AK, Wabwire FM. Epidemiology of HPV genotypes in Uganda and the role of the current preventive vaccines: A systematic review. Infect Agent Cancer. 2011;6:11

10. Ministry of health Uganda \& ORC Macro 2006. Uganda HIV/AIDS serobehavioral survey 2004-2005. Available at http://dhsprogram.com/pubs/pdf/ ais2/ais2.pdf. Accessed 1 Nov 2013.

11. Ministry of Health Uganda, ICF, CDC, USAID \& WHO 2012. Uganda AIDS indicator Survey 2011. Available at http://dhsprogram.com/pubs/pdf/AlS10/ Als10.pdf. Accessed 1 Nov 2013.

12. Rysavy MB, Kresowik JDK, Liu D, Mains L, Lessard M, Ryan GL. Human Papillomavirus Vaccination and Sexual Behavior in Young Women. J Pediatr Adolesc Gynecol. 2014;27:67e71.

13. Marchand E, Glenn BA, Bastani R. HPV Vaccination and Sexual Behavior in a Community College Sample. J Community Health. 2013:38(6). doi:10.1007/ s10900-013-9710-0.

14. Mattebo M, Grün N, Rosenblad A, Larsson M, Häggström-Nordin E, Dalianis $T$, et al. Sexual experiences in relation to HPV vaccination status in female high school students in Sweden. Eur. J. Contracept. Reprod. Health Care. 2014;19:86-92.

15. Aujo JC, Bakeera-Kitaka S, Kiguli S, Mirembe F. No difference in sexual behavior of adolescent young women following Human Papilloma Virus vaccination: a case study two districts in Uganda; Nakasongola and Luwero. BMC Public Health. 2014;14:155. doi:10.1186/1471-2458-14-155.

16. Smith LM, Kaufman JS, Strumpf EC, Lévesque LE. Effect of human papillomavirus (HPV) vaccination on clinical indicators of sexual behaviour among adolescent girls: the Ontario Grade 8 HPV Vaccine Cohort Study. CMAJ. 2015;187(2):E74-81. doi:10.1503/cmaj.140900. Epub 2014 Dec 8

17. Jena $A B$, Goldman DP, Seabury SA. Incidence of sexually transmitted infections after human papillomavirus vaccination among adolescent females. JAMA Intern Med. 2015:175(4):617-23. doi:10.1001/jamainternmed.2014.7886.

18. Mayhew A, Kowalczyk Mullins TL, Ding L, Rosenthal SL, Zimet GD, Morrow C, et al. Risk Perceptionss and Subsequent Sexual Behaviors After HPV Vaccination in Adolescents. Pediatrics. 2014;133:404; originally published online February 2, 2014. doi:10.1542/peds.2013-2822.

19. Brewer NT, Fazekas Kl. Predictors of HPV vaccine acceptability: a theoryinformed, systematic review. Prev Med. 2007;45:107.

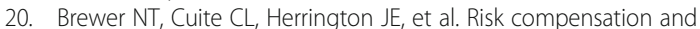
vaccination: can getting vaccinated cause people to engage in risky behaviors? Ann Behav Med. 2007;34:95

21. Brabin L, Roberts SA, Stretch R, et al. A survey of adolescent experiences of human papillomavirus vaccination in the Manchester study. Br J Cancer. 2009;101(9):1502-4.
22. Mather T, McCaffery K, Juraskova I. Does HPV vaccination affect women's attitudes to cervical cancer screening and safe sexual behavior? Vaccine. 2012;30:3196-201

23. Ruiz-Sternberg ÁM, Pinzón-Rondón ÁM. Risk perceptions and sexual behavior in HPV-vaccinated and unvaccinated young Colombian women. Int J Gynecol Obstet. 2014;126:205-8.

24. Bednarczyk RA, Davis R, Ault K, et al. Sexual activity-related outcomes after human papillomavirus vaccination of 11- to 12-year-olds. Pediatrics. 2012; 130:798.

25. LaMontagne DS, Barge S, Le NT, Mugisha E, Penny ME, Gandhi S, et al. Human papillomavirus vaccine delivery strategies that achieved high coverage in low-and middle income countries. Bull World Health Organ. 2011;89:821-830B. doi:10.2471/BLT.11.089862.

26. Altman DG. Practical statistics for medical research. London: Chapman \& Hall; 1991

27. Banura C, Franceschi S, Doorn LJ, Arslan A, Wabwire-Mangen F, Mbidde EK, et al. Infection with human papillomavirus and HIV among young women in Kampala, Uganda. J Infect Dis. 2008;197(4):555-62.

28. Downing RG, Otten RA, Marum E, et al. Optimizing the delivery of HIV counseling and testing services: the Uganda experience using rapid HIV antibody test algorithms. J Acquir Immune Defic SyndrHumRetrovirol. 1998; 18:384-8.

\section{Submit your next manuscript to BioMed Central and we will help you at every step:}

- We accept pre-submission inquiries

- Our selector tool helps you to find the most relevant journal

- We provide round the clock customer support

- Convenient online submission

- Thorough peer review

- Inclusion in PubMed and all major indexing services

- Maximum visibility for your research

Submit your manuscript at www.biomedcentral.com/submit

) Biomed Central 